\title{
BMJ Open Predictive value of cell-surface markers in infections in critically ill patients: protocol for an observational study (ImmuNe FailurE in Critical Therapy (INFECT) Study)
}

Andrew Conway Morris, ${ }^{1,2}$ Deepankar Datta, ${ }^{2,3}$ Manu Shankar-Hari, ${ }^{4}$ Christopher J Weir, ${ }^{5}$ Jillian Rennie, ${ }^{2}$ Jean Antonelli, ${ }^{6}$ Adriano G Rossi, ${ }^{2}$ Noel Warner, ${ }^{7}$ Jim Keenan, ${ }^{7}$ Alice Wang, ${ }^{7}$ K Alun Brown, ${ }^{8}$ Sion Lewis, ${ }^{8}$ Tracey Mare, ${ }^{8}$ A John Simpson, ${ }^{9}$ Gillian Hulme, ${ }^{10}$ Ian Dimmick, ${ }^{10}$ Timothy S Walsh ${ }^{2,3}$

To cite: Conway Morris A, Datta D, Shankar-Hari M, et al. Predictive value of cellsurface markers in infections in critically ill patients: protocol for an observational study (ImmuNe FailurE in Critical Therapy (INFECT) Study). BMJ Open 2016;6: e011326. doi:10.1136/ bmjopen-2016-011326

- Prepublication history and additional material is available. To view please visit the journal (http://dx.doi.org/ 10.1136/bmjopen-2016011326)

$K A B$ and ID have retired, affiliations are those at the time of protocol drafting.

Received 28 January 2016 Revised 14 April 2016 Accepted 9 June 2016

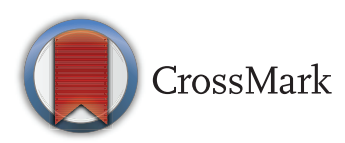

For numbered affiliations see end of article.

Correspondence to Dr Andrew Conway Morris; mozza@doctors.org.uk

\section{ABSTRACT}

Introduction: Critically ill patients are at high risk of nosocomial infections, with between $20 \%$ and $40 \%$ of patients admitted to the intensive care unit (ICU) acquiring infections. These infections result in increased antibiotic use, and are associated with morbidity and mortality. Although critical illness is classically associated with hyperinflammation, the high rates of nosocomial infection argue for an importance of effect of impaired immunity. Our group recently demonstrated that a combination of 3 measures of immune cell function (namely neutrophil CD88, monocyte HLA-DR and \% regulatory $T$ cells) identified a patient population with a 2.4-5-fold greater risk for susceptibility to nosocomial infections.

Methods and analysis: This is a prospective, observational study to determine whether previously identified markers of susceptibility to nosocomial infection can be validated in a multicentre population, as well as testing several novel markers which may improve the risk of nosocomial infection prediction. Blood samples from critically ill patients (those admitted to the ICU for at least 48 hours and requiring mechanical ventilation alone or support of 2 or more organ systems) are taken and undergo whole blood staining for a range of immune cell surface markers. These samples undergo analysis on a standardised flow cytometry platform. Patients are followed up to determine whether they develop nosocomial infection. Infections need to meet strict prespecified criteria based on international guidelines; where these criteria are not met, an adjudication panel of experienced intensivists is asked to rule on the presence of infection. Secondary outcomes will be death from severe infection (sepsis) and change in organ failure.

Ethics and dissemination: Ethical approval including the involvement of adults lacking capacity has been obtained from respective English and Scottish Ethics Committees. Results will be disseminated through presentations at scientific meetings and publications in peer-reviewed journals.

Trial registration number: NCT02186522; Pre-results.

\section{Strengths and limitations of this study}

Multi-site study recruiting from geographically and clinically diverse populations.

- Multisite nature has produced a programme of flow cytometry standardisation which we believe to be both robust and reproducible and so sets the scene for potential clinical use of these assays should they prove to be of value.

- We have taken steps to try to minimise variability in the diagnosis of infection, through the use of rigid criteria and consensus review of cases which do not meet these criteria.

- The weaknesses of this study are that it is observational, and thus will not be able to inform clinicians of what actions they should take in response to these results should clinically useable tests be developed.

\section{INTRODUCTION}

Critical illness increases the risk of nosocomial infection, with between $20 \%$ and $40 \%$ of patients admitted to the intensive care unit (ICU) acquiring infections during their critical care stay, ${ }^{12}$ a rate that approaches that seen in haematopoietic stem cell transplantation. $^{3}$ Provision of organ support requires the disruption of epithelial and mucosal barrier innate immune system protection through the placement of devices such as endotracheal tubes, urinary catheters and central venous catheters. These infections are often bacterial and are associated with increased antibiotic use. ${ }^{12}$ In addition to bacterial infections, critically ill patients are at risk of reactivation of latent viral infections. ${ }^{4}$ Therefore, it is thought that the combination of immune vulnerability and microbial colonisation is responsible for the 
high rates of nosocomial infection seen in critically ill patients. $^{1}$

Critical illness resulting from trauma, sepsis and postsurgical complications all have commonality in the innate and adaptive immune responses. ${ }^{56}$ Many diseases that can precipitate the need for exogenous organ support and admission to intensive care are characterised by a profound systemic inflammatory response, ${ }^{7}$ with associated immune cell activation ${ }^{8}$ and immune system-mediated organ damage. ${ }^{9}$ However, it is now increasingly apparent that this overexuberant inflammation is accompanied by an equally vigorous counterregulatory anti-inflammatory response. ${ }^{5}{ }^{10}$ The apparently maladaptive, complex immune dysfunction in critically ill patients manifests across a range of cellular actions and functions, involving both the innate and adaptive arms of the immune system..$^{510-15}$ Defects have been noted in neutrophils, ${ }^{11}$ monocytes, ${ }^{12}{ }^{16} 17$ T lymphocytes $^{10}{ }^{13-15}$ and splenic dendritic cells. ${ }^{10}$ The recent identification of elevated proportions of regulatory helper-T cells (Tregs) in sepsis ${ }^{1415}$ is in keeping with the supposition that much of the immunosuppression arises from the overactivation of counter-regulatory mechanisms. In human and experimental sepsis, Tregs mediate lymphocyte anergy and are associated with worse outcomes. ${ }^{15}$

\section{Rationale for the study}

Although it seems plausible that the immune defects found in critical illness are associated with an increased risk of acquiring nosocomial infections, the concept that immune cell abnormalities always precede nosocomial infections, necessary for causality, is inconsistently reported in the literature. ${ }^{14}{ }^{16}$ Following our previous study, ${ }^{11}$ which demonstrated the benefits of combining measures of immune dysfunction on predicting nosocomial infection, there is a need to validate the markers in a new cohort. Undertaking this as a multicentre study will help develop standardised flow cytometric measures and improve the external validity of the markers that predict risk of nosocomial infection. If a selected set of immune abnormalities that could be modified with a single intervention are present together, then development of a test could stratify patients for risk of nosocomial infections for targeted interventions. ${ }^{17}$ Finally, identifying multiple cell defects/markers will help design future trials of therapies and prophylactic measures to prevent nosocomial infections.

\section{Hypotheses}

1. Markers of neutrophil, monocyte and T-cell hypoactivity will predict those critically ill patients who are at risk of nosocomial infection.

2. These markers will have added predictive value when combined.

3. Additional predictive ability will be achieved through examination of monocyte and Treg subsets and other cell surface markers of immune cell function.

\section{Primary aims}

1. To validate the ability of neutrophil CD88, monocyte HLA-DR and percentage of Tregs to predict those patients at risk of nosocomial infection.

2. To determine the optimal way of combining these measures to risk stratify patients.

3. To develop a clinically useable test.

\section{Secondary aims}

1. To determine the relationship between the measures outlined above and risk of death from sepsis.

2. To determine whether more detailed phenotyping of monocyte and Treg subsets provides additional value in predicting risk of nosocomial infection.

3. To explore whether other cell surface measures of immune cell function and phenotype may predict nosocomial infection.

\section{METHODS AND ANALYSIS}

This protocol outlines a multicentre, prospective observational study in which critically ill patients will be recruited and assessments made of immune cell surface phenotypic markers at multiple time points. Patients will be followed to determine outcomes of interest, the primary outcome being development of nosocomial infection.

\section{Study population}

The population will be drawn from four UK adult ICUs, consisting of:

1. Royal Infirmary, Edinburgh (liver/general unit);

2. Western Infirmary, Edinburgh (neurosciences/ general unit);

3. St Thomas' Hospital, London (cardiac/general unit);

4. Sunderland Royal Hospital (general unit).

Consecutive patients will be eligible if they are aged $>16$ (Scotland) or $>18$ (England), receiving support of level 3 care (ie, requiring invasive support of respiratory system alone, or two or more other organ systems (haemofiltration, inotropes/vasopressors)) and predicted to remain in the ICU for at least 48 hours.

Exclusion criteria are not being expected to survive for a further 24 hours, known or suspected ICU-acquired infection at time of screening (non-ICU-acquired nosocomial infection-ie, non-ICU healthcare-associated infection is not an exclusion), known inborn errors of immune function, immunosuppression (corticosteroids up to $400 \mathrm{mg}$ hydrocortisone equivalent daily dose permitted), HIV infection, hepatitis B and $\mathrm{C}$ infection, receiving extracorporeal membrane oxygenation, pregnancy and previously enrolment in the study.

Co-enrolment is permitted where the overall phlebotomy burden on patients is acceptable $(<50 \mathrm{~mL}$ at any one time point and total phlebotomy load of $<150 \mathrm{~mL}$ ), where the co-enrolled study is deemed unlikely to affect the primary end point of the ImmuNe FailurE in Critical Therapy (INFECT) study and where a formal coenrolment agreement is in place. 


\section{SAMIPLING SCHEDULE}

Blood samples for flow cytometric analysis of surface receptor expression will be taken on study enrolment, and then on day 2 after study enrolment, and at 48-hour intervals until day 12, a maximum of seven samples per patient. Patients discharged from the ICU within the 16-day study window will have a maximum of two further samples taken at 3-4-day intervals up to day 12 of the study to minimise burden of venepuncture. Where possible, these will be collected at the time of routine venepuncture for clinical sampling. Patients will be followed for the development of infection for 16 days (by this time, the great majority of infections had been acquired in the original study and few patients were left in the ICU alive and without infection). All survivors who remain in hospital will be followed to this point including those who have left the ICU.

Clinical and demographic data will be collected including age, sex, functional comorbidity index, smoking status, physiological data (cute Physiology and Chronic Health Evaluation score II (APACHE II) score on admission, Sequential Organ Failure Assessment (SOFA) score at baseline, full blood count and differential white cell count) and clinical data (admission diagnosis, admission source, antibiotics, invasive devices present and duration). Similar clinical data will be collected at each sample time point. Hospital outcome (ie, discharged alive, died or transferred to another hospital) will also be collected.

\section{DEFINITION OF INFECTION}

Infections will need to meet strict, predefined criteria (see online supplementary appendix A) and will be assessed by research staff blinded to the immune phenotyping data. Suspected infections which do not meet the criteria for confirmed infection will be reviewed 'offline' by a panel of clinicians blinded to the immune phenotyping data using information from a prospectively collected proforma. The outcomes from this will be 'highly likely' infection and 'unlikely infection/colonisation' (colonisation being where microbes are grown in the absence of evidence for infection). The day infection is acquired will be defined as the day the sample which shows positive for microbiology was taken. In the case of sterile cultures where the clinician strongly suspects infection, the day of infection will be defined as the day strong clinical suspicion was raised. Therefore, outcomes will be (1) 'confirmed', (2) 'probable infection' and (3) 'unlikely infection/colonisation'.

Patients who are transferred to a non-study hospital will have data included up until the day of discharge and will be followed up via telephone contact from the recording unit to ascertain whether there were any confirmed or suspected infections in the days following transfer (up to 16 days poststudy entry). Patients transferred from one participating site to another will remain in the study and have data and samples collected as per study protocol.
FLOW CYTOMETRIC STANDARDISATION AND SAMPLE

\section{STAINING}

All sites have standardised on the same platform the FACS Canto II (Becton Dickinson Biosciences, San Jose, California, USA-from here on BDB) for flow cytometric analysis of samples. Machines will be standardised by monthly matching of target values using a common batch of Cytometry Setup and Tracking (CS\&T) beads (BDB), and daily internal quality control using CS\&T beads.

Leucocyte cell surface staining will be conducted using antibodies supplied by BDB. All sites use antibodies from the same batch. Staining, data capture and storage will be conducted in accordance with a single study standard operating procedure.

Flow cytometry data will be held centrally. Final analysis will be conducted using a single analysis platform, VenturiOne (Applied Cytometry, Sheffield, UK).

\section{CELLULAR IMIMUNOPHENOTYPING}

The primary measures are to validate our previous findings, ${ }^{11}$ namely: neutrophil CD88, monocyte HLA-DR and percentage CD4 cells expressing the CD25+/CD127lo regulatory phenotype, using the cut-offs defined by our previous study. ${ }^{11}$

Additional phenotypic measurements include neutrophil and monocyte activation markers (including CD11b, CD66b, CD312), subtypes of regulatory T cells and Treg activation, frequency of monocyte subsets and subset HLA-DR expression, frequency of dendritic cell subtypes, expression of monocyte and lymphocyte PD1 and PDL1, frequency of granulocytic and monocytic myeloid-derived suppressor cells, and frequency of B-cell subtypes.

\section{SAMPLE SIZE}

In a cohort studied previously, ${ }^{11} 34 \%$ of patients were confirmed as having secondary sepsis. We therefore expect the panel of markers to perform well when predicting $25-45 \%$ of patients to have secondary sepsis. Across a broad range of possible positive predictive value (PPV) performance (50-90\%), the 95\% CI width for the PPV would range from $\pm 5.5 \%$ to $\pm 15.9 \%$, indicating moderately precise estimation of the PPV. Using a similar rationale, we would expect the $95 \%$ CI width for the negative predictive value (NPV) to range from $\pm 4.3 \%$ to $\pm 10.8 \%$, indicating precise estimation of the NPV. A conservative estimate of a $50 \%$ PPV with a $35 \%$ rate of secondary sepsis would yield a $95 \%$ CI of $39-61 \%$ in a cohort of 200 patients. We also intend to recruit 20 patients initially to confirm that the reformulated flow cytometric tests match the performance of those from the derivation cohort, ${ }^{11}$ and thus we propose recruiting to a 220 patient cohort.

\section{INFORMED CONSENT}

Consent and assent procedures will be conducted under the relevant legislation, in England (Mental Capacity 
Act, 2005) or Scotland (Adults with Incapacity (Scotland) Act, 2000) for consent/assent of adults without capacity. In England, assent is obtained, where possible, following discussion with the patient's next of kin (personal consultee). Where a personal consultee is unavailable, assent is provided by a professional consultee, being a senior medical professional who is not in the research team. In circumstances where next of kin are unable to attend the ICU promptly, deferred consent procedures are used.

In Scotland, the patient's relative or welfare attorney provides consent. If the patient's relative or welfare attorney is unable to attend the ICU, consent may be provided in a telephone conversation providing a second member of staff witnesses the discussion.

Patients who recover capacity will be approached to provide retrospective consent.

\section{SAFETY OF PARTICIPANTS}

The only safety concern is that of potential additional venepuncture in patients; the risks of this are of minor harm (bruising). Post-ICU sampling (where indwelling lines are not present for blood sampling) is limited to a maximum of two samples.

\section{DATA ANALYSIS PLAN}

A detailed statistical analysis plan will be finalised prior to the locking of the study database.

Studies will be conducted to ensure that the flow cytometric readings of each marker are reproducible to demonstrate they can be a useful test. Interobserver and intraobserver reliability studies will be conducted with three expert readers of the data. Fifty data files will be read to determine interobserver agreement, with 13 files for intraobserver agreement. After a preliminary reliability study, an optimisation stage will occur with expert meetings to further improve flow cytometric gating strategies in problematic markers to ensure that we do not wrongly reject markers. A second reliability study will then be conducted using the same sample size to select markers taken forward to future stages of analysis.

The main analysis of the primary outcome will be an analysis of the PPV and NPV of immune dysfunctions in predicting nosocomial infection, as well as the predictive ability of combinations of immune dysfunction. The primary analysis will include both 'confirmed' and 'probable' infections as 'infections', with analysis by 'confirmed' infections only conducted as a sensitivity analysis. Sensitivity and specificity will also be determined. Ninety-five per cent CIs will be calculated for all measures of predictive accuracy.

As with the derivation cohort, ${ }^{6}$ 'immune function/dysfunction' will be defined by the time point before first nosocomial infection occurs, censored for 2 days prior to infection.

As a secondary analysis, a Cox model of time to acquisition of infection will be fitted, with the classification 'immune dysfunction' or 'no dysfunction' as one independent variable. The other independent variables will be potential clinical confounders identified in previous epidemiological work looking at risk factors for nosocomial infection ${ }^{1}{ }^{2}$ and will demonstrate whether our novel tests add predictive value over routinely available clinical and demographic data.

The association of immune dysfunctions with the secondary outcome measures ICU outcome (lived/died) and death from sepsis (yes/no) will be assessed using the same methods as the main analysis of the primary outcome.

Determination of the clinical utility of the test will come from a two-stage assessment. First, the reliability of the flow cytometric markers will be assessed, and markers for clinical use must have excellent inter-rater and intra-rater reliability. Second, the markers must be clinically valuable, adding predictive ability beyond that which can be gained from clinical assessment and standard laboratory parameters. Both these assessments are inherent in the data analysis programme outlined above, and will be reviewed both internally by the study consortium and also by a group of key clinical stakeholders who are independent of the study consortium. Alongside this, a mocked-up clinical workflow will be developed based on the laboratory procedures developed to run this study, using the experience of Becton Dickinson's established clinical assay systems.

\section{ETHICS AND DISSEMINATION}

Local research governance approval has been granted by the three National Health Service (NHS) organisations covering the four sites where the study is being conducted.

\section{Study management}

The study is managed by the Edinburgh Clinical Trials Unit (ECTU) and the Edinburgh Critical Care Research Group (ECCRG) at the University of Edinburgh, Edinburgh, Scotland.

\section{Sponsorship}

The study is co-sponsored by the University of Edinburgh and NHS Lothian.

\section{Duration of study}

The study was planned to run for 3 years, starting October 2012, with initial assay development leading to patient recruitment starting July 2014. Following successful requests for grant extension due to delays in setting up recruiting sites, the expected end date for recruitment is 31 January 2016. Flow cytometric data will require postacquisition processing prior to analysis; this is expected to be completed by 1 April 2016. We expect to have completed our primary data analysis with a view to dissemination of results by August 2016, and at this point we will consider the study complete. 
Results from the study will be reported according to the Standards for Reporting of Diagnostic Accuracy $(\mathrm{STARD}){ }^{18}$ guidelines for reporting diagnostic accuracy studies and disseminated via presentations at scientific meetings and publications in peer-reviewed journals.

\section{Author affiliations}

'University Division of Anaesthesia, Department of Medicine, Addenbrooke's Hospital, Cambridge, UK

${ }^{2}$ MRC Centre for Inflammation Research, University of Edinburgh, Edinburgh, UK

${ }^{3}$ University of Edinburgh School of Clinical Sciences, Edinburgh Critical Care Research Group, Edinburgh, UK

${ }^{4}$ Intensive Care Unit, Guy's and St Thomas' Hospital NHS Foundation Trust, London, UK

${ }^{5}$ Edinburgh Health Services Research Unit, Centre for Population Health

Sciences, University of Edinburgh, Edinburgh, UK

${ }^{6}$ Edinburgh Clinical Trials Unit, University of Edinburgh, Western General Hospital, Edinburgh, UK

${ }^{7}$ Becton Dickinson Bioscience, Franklin Lakes, New Jersey, USA

${ }^{8}$ Vascular Immunology Research Laboratory, Rayne Institute (King's College London), St Thomas' Hospital, London, UK

${ }^{9}$ Institute of Cellular Medicine, Newcastle University, Newcastle upon Tyne, UK ${ }^{10}$ Flow Cytometry Core Facility Laboratory, Faculty of Medical Sciences, Centre for Life, Newcastle University, Newcastle upon Tyne, UK

Twitter Follow Andrew Conway Morris at @andymoz78, Deepankar Datta at @deepankardatta and Manu Shankar-Hari at @msh_manu

Acknowledgements The authors wish to acknowledge the contribution of Sharon Cookson of Northumbria University and Dr Andrew Filby, Newcastle University for assistance with flow cytometry analysis, Dr Alasdair Roy of Sunderland Royal Hospital and Dr Anthony Bateman of Western General Hospital, Edinburgh for their assistance with establishing these research sites.

Contributors TSW, NW, KAB and ACM were involved in study conception. TSW, NW, JK, KAB, JS and ACM were involved in obtaining funding. JR, AW, AGR, SL, TM, GH and ID were involved in development of flow cytometry techniques. TSW, DD, CJW and ACM were involved in development of the statistical analysis plan. TSW, DD, JA, AGR, NW, JK, MS-H, KAB, JS, ID and $A C M$ were involved in protocol development. $D D$ and $A C M$ were involved in manuscript drafting. All authors have reviewed and approve the final version.

Funding The study is funded by a grant from Innovate UK (formerly Technology Strategy Board), grant number 15457-108136 in conjunction with Becton Dickinson bioscience. CJW is also supported in this work by NHS Lothian via the Edinburgh Health Services Research Unit. ACM is also supported in this work by a grant from the National Institute of Academic Anaesthesia for the investigation of the role of Tregs in sepsis.

Competing interests None declared.

Ethics approval The study has been approved by the Scotland A Research Ethics Committee (13/SS/0022) for Scottish sites, and the Warwick and Coventry Research Ethics Committee (13/WM/0207) for English sites.

Provenance and peer review Not commissioned; externally peer reviewed.

Data sharing statement Unpublished data will be available from the corresponding author by direct application.
Open Access This is an Open Access article distributed in accordance with the Creative Commons Attribution Non Commercial (CC BY-NC 4.0) license, which permits others to distribute, remix, adapt, build upon this work noncommercially, and license their derivative works on different terms, provided the original work is properly cited and the use is non-commercial. See: http:// creativecommons.org/licenses/by-nc/4.0/

\section{REFERENCES}

1. Vincent JL, Rello J, Marshall J, et al. International study of the prevalence and outcomes of infection in intensive care units. JAMA 2009;302:2323-9.

2. Vincent JL, Bihari DJ, Suter PM, et al. The prevalence of nosocomial infection in intensive care units in Europe. Results of the European Prevalence of Infection in Intensive Care (EPIC) Study. JAMA 1995;274:639-44.

3. Mendes ET, Dulley F, Basso M, et al. Healthcare-associated infection in hematopoietic stem cell transplantation patients: risk factors and impact on outcome. Int J Infect Dis 2012;16:e424-8.

4. Limaye AP, Kirby KA, Rubenfeld GD, et al. Cytomegalovirus reactivation in critically ill immunocompetent patients. JAMA 2008;300:413-21.

5. Hotchkiss RS, Monneret G, Payen D. Sepsis-induced immunosuppression: from cellular dysfunctions to immunotherapy. Nat Rev Immunol 2013;13:862-74.

6. Xiao W, Mindrinos MN, Seok J, et al. A genomic storm in critically injured humans. J Exp Med 2011;208:2581-90.

7. Adib-conquy M, Cavaillon JM. Stress molecules in sepsis and systemic inflammatory response syndrome. FEBS Lett 2007;581:3723-33.

8. Sakamoto $\mathrm{Y}$, Mashiko $\mathrm{K}$, Matsumoto $\mathrm{H}$, et al. Systemic inflammatory response syndrome score at admission predicts injury severity, organ damage and serum neutrophil elastase production in trauma patients. J Nihon Med Sch 2010;77:138-44.

9. Muller Kobold AC, Tulleken JE, Zijlstra JG, et al. Leukocyte activation in sepsis; correlations with disease state and mortality. Intensive Care Med 2000;26:883-92.

10. Boomer JS, To K, Chang KC, et al. Immunosuppression in patients who die of sepsis and multiple organ failure. JAMA 2011;21:2594-605.

11. Conway Morris A, Kefala K, Wilkinson TS, et al. C5a mediates peripheral blood neutrophil dysfunction in critically ill patients. Am J Respir Crit Care Med 2009;180:19-28.

12. Döcke WD, Randow F, Syrbe U, et al. Monocyte deactivation in septic patients: restoration by IFN-gamma treatment. Nat Med 1997;3:678-81.

13. Venet F, Chung CS, Monneret G, et al. Regulatory T cell populations in sepsis and trauma. J Leukoc Biol 2008;83:523-35.

14. Conway Morris A, Anderson N, Brittan M, et al. Combined dysfunctions of immune cells predict nosocomial infection in critically ill patients. Br J Anaesth 2013;111:778-87.

15. Venet $\mathrm{F}$, Chung $\mathrm{CS}$, Kherouf $\mathrm{H}$, et al. Increased circulating regulatory $\mathrm{T}$ cells $(\mathrm{CD} 4(+) \mathrm{CD} 25(+) \mathrm{CD} 127(-))$ contribute to lymphocyte anergy in septic shock patients. Intensive Care Med 2009;35:678-86.

16. Lukaszewicz AC, Grienay M, Resche-Rigon M, et al. Monocytic HLA-DR expression in intensive care patients: interest for prognosis and secondary infection prediction. Crit Care Med 2009;37:2746-52.

17. Meisel C, Schefold JC, Pschowski R, et al. Granulocyte-macrophage colony-stimulating factor to reverse sepsis-associated immunosuppression: a double-blind, randomized, placebo-controlled multicenter trial. Am J Respir Crit Care Med 2009;180:640-8.

18. Bossuyt PM, Reitsma JB, Bruns DE, et al. STARD 2015: an updated list of essential items for reporting diagnostic accuracy studies. BMJ 2015;351:h5527. 